over a three-year period. Audiograms were made to all employees, at the same time with official determination of the noise level, carried out by the Public Health Authority of the county. In selected cases, the workers were also led to specialised examinations (cardiology, neurological, psychiatric, ENT).

Results Official noise level determinations have shown overtaking in many of the technological process points (ironing presses, buttonhole machines, edge banding, staple machines etc.). In situations where workers were rotated at short intervals (30 days), they showed minimal symptoms of high-loud exposure. In situations where workers had fixed workstations, after three years of exposure, a decrease with $5-10 \mathrm{~dB}(\mathrm{~A})$ in auditory acuity was observed, associated with sleep disturbances, nervousness and irritability, a slight increase in blood pressure, at about $20 \%$ of the employees (especially if they were over 50 years old and worked in the noise before). During week-ends and holidays, everyone was feeling good.

Discussion The textile industry is an important sector of concentration of professional high-noise exposure pathology. Technical and organisational measures (risk recognition, wearing individual hearing protection equipment, resting in quiet rooms, separation of noise-generating technology processes, high-tech equipment), as well as medical ones (decrease in headphones use, health education, thorough medical checks), lead to better management of the negative impact of human health noise.

\section{FEELING STRAINED BY THE EXPECTATION TO BE AVAILABLE - GENDER DIFFERENCES OF EMPLOYEES IN SMALL AND MEDIUM-SIZED ENTERPRISES}

A Voss* ${ }^{*}$ W Fischmann, N Amler, E Wischlitzki, J Kiesel, H Drexler. Friedrich-AlexanderUniversität Erlangen-Nürnberg, Germany

\subsection{6/oemed-2018-ICOHabstracts. 1522}

Introduction The increasing use of digital media such as mobile phone, e-mail, messenger services etc. enable a permanent availability of employees, which may lead to mental strain. This study focuses on possible gender-specific differences in feeling strained by the expectation to be permanently available for colleagues, superiors, or customers.

Methods A psychological risk assessment has been carried out with 933 employees within the framework of a collaborative project ('RegioKMUnet'), funded by the German Federal Ministry of Education and Research. Data were collected from October 2016 to June 2017 in 10 small and medium-sized enterprises by paper and online surveys. The basic question was if the respondents had to be short-termed available during their free time or working time and whether that led to mental strain. Data were analysed using IBM SPSS 24.

Results The sample consisted of 569 male and 364 female respondents. $85.6 \%$ of the males and $80.1 \%$ of the females reported they had to be available during their working time. The expected availability led to mental strain for $59.2 \%$ of the men and $50.3 \%$ of the women. During free time $32 \%$ males and $17.5 \%$ females stated they had to be available. For $51.8 \%$ of the men and $37.5 \%$ of the women this led to a feeling of being strained.

A chi-square test confirmed the gender difference of strain during free time $(p=0.042)$.
Conclusion In general the mental strain caused by the expectation of availability is stronger for men, with a significantly higher share during working time. In the free time the distance is even greater but inconclusive due to low case numbers. Additional research is needed to clarify if mental strain caused by availability is confirmedly greater for men. Furthermore possible influence by part-time and full-time working as well as the kind of work activity should be considered.

\section{BREAST FEEDING PRACTICES AMONG WORKING MOTHERS ATTENDING 'POSTNATAL' CLINICS IN TWO HOSPITAL IN KAMPALA, UGANDA}

Khayongo Barbra Clara*. Ministry of Gender, Labour and Social Development, Kampala, Uganda International Commission on Occupational Healt, Allied Health Workers' Association, Kampala, Uganda, Uganda Community and Occupational Health Association

\subsection{6/oemed-2018-ICOHabstracts. 1523}

Introduction Breast milk is critical for proper development of an infant during the first two years of life. The increased rate of women's participation in the labour force has resulted in a 'double work day' which results in a burden of having to accomplish both reproductive and productive roles. The Employment Act 2006, maternity leave is 12 weeks. This is insufficient as it's below recommended 6 months of exclusive breastfeeding thus maternity protection is left to the discretion of the employer and breastfeeding working mothers.

Method A mixed study design using both quantitative and qualitative data collection and analysis methods was used.

Results 232 questionnaires were administered to working mothers attending 'postnatal clinics. 23 KI guides were administered to key decision managers purposively selected from 23 institutions where primary respondents worked. Whereas 224 (96.5\%) were granted maternity leave a proportion of 8 $(3.5 \%)$ were not granted maternity leave. During day time, on demand breastfeeding (over 10 times) was practiced by 225 $(97 \%)$ respondents before resuming full time work. This reduced drastically on resuming full time work to half (between 5 to 7 times during the day) for those in selfemployed and service sectors and only twice for those in Government Ministries, Departments, Agencies, NGO, Schools and banks. $53(22.8 \%)$ breastfed their babies at workplace of these $46(86.8 \%)$ said that the babies were simply laid within working station not designated as a workplace day care centre.

Discussion Whereas we continue to advocate for exclusive breastfeeding, we need to consider the effect of productive roles experienced by working mothers who resume work before their babies are 6 months thus interrupt breastfeeding pattern. Work places in informal and formal sectors had no provisions for nursing mothers to care for their babies nor express their breast milk.

\section{HOUSEWIVES AND MEXICAN WOMEN AT WORK: HEALTH AND PSYCHOLOGICAL FACTORS}

L Fernando Arias-Galicia*. Centro de Investigaciones Interdisciplinarias en Psicología, Universidad Autónoma del Estado de Morelos

10.1136/oemed-2018-ICOHabstracts. 1524 
Introduction An important group of working women has been laid aside of research: housewives and micro entrepreneurs. Perhaps multiple roles are detrimental to housewives' both physical and mental health; it is supposed that these damaging effects are worst in the case of women taking care of housekeeping tasks and being workers at the same time. Therefore: how housewives, female microentrepreneurs, workers and professionals compare in the above mentioned fields?

Methods 353 women were surveyed in Cuernavaca, Mexico. $25 \%$ were housewives, $21 \%$ were housewives which sold goods or rendered services (baby sitting, laundry, ironing, for instance) to people outside the family circle, $13 \%$ were housewives and micro entrepreneurs at the same time, 5\% said were blue-collar workers and housewives, 7\% saw themselves as clerks and house-wives, $21 \%$ were professionals and housewives, and 5\% saw themselves as professionals without being housewives at the same time.

The BREF-WHOQOL, the Siegrist Effort-Reward Questionnaire (slightly modified to adapt it to the sample), a stress, and self-efficacy, self-esteem, sense of life as well as a demographic questionnaire were administered.

Results MANOVAS were calculated for the whole sample. Housewives rendered the lowest scores in mental health and self-efficacy, followed by micro-entrepreneurs, and manual workers. Regarding mental health, office employees, professionals and women without being housewives were better off as well as considering self-esteem, physical health, stress and recognition from their families. Stress correlated egatively with family satisfaction, recognition from their family, physical health, self-efficacy, self-esteem, and mental health. Effort was positively associated with stress, and negatively with the rest of variables. However, from a practical viewpoint scores were minor, mounting to less than a point.

Conclusion Protective factors balance negative ones. Public policies should aim to pay attention to housewives since their important role within families impact in children and husbands as well as to society.

\section{Work and Vision}

\section{WORK AND VISION}

${ }^{1}$ Miguel S Kabilio*, ${ }^{2}$ Agueda Muñoz del Carpio Toia. ${ }^{1}$ Australia and New Zealand Society of Occupational Medicine (ANZSOM), Perth, Australia; ${ }^{2}$ Universidad Catolica de Santa Maria, Arequipa, Peru

\subsection{6/oemed-2018-ICOHabstracts. 1525}

Aim of special session Investigate, diagnose work-related and environment-related ocular conditions

Apply appropriate ergonomic work conditions for reducing occupational asthenopia.

Promote healthy work lighting

${ }^{1}$ Prof Bruno Pisaniello, ${ }^{2}$ Dr Peder Wolkoff, ${ }^{3}$ Prof Bruno Piccoli

${ }^{1}$ School of Public Health, University of Adelaide, South Australia, Australia

${ }^{2}$ National research Centre for the Working Environment, Copenhagen, Denmark

${ }^{3}$ Institute of Public Health - Section of Occupational Health, Catholic University of the Sacred Heart, Roma, Italy

\section{8a OFFICE LIGHTING SURVEYS - REFOCUSSING ON THE} EYE AND HEALTH

${ }^{1}$ Dino Pisaniello*, 'Sharyn Gaskin, ${ }^{2}$ Marco D'Orso, ${ }^{3}$ Bruno Piccoli. 'School of Public Health, University of Adelaide, Adelaide, Australia; ${ }^{2}$ School of Medicine, University of Milan Bicocca, Monza, Italy; ${ }^{3}$ Institute of Public Health - Section of Occupational Health, Catholic University of the Sacred Heart, Rome, Italy

\subsection{6/oemed-2018-ICOHabstracts. 1526}

Introduction Current guidance on office lighting tends to be oriented towards illumination engineering, i.e. achieving a certain lighting environment. The selection of lamps and luminaires from a vast array of current lighting options, as well as their layout, often reflects architectural style and energy efficiency, rather than 'healthy' lighting - which aims to eliminate or minimise adverse health effects, including visual discomfort, arising from visual tasks and ambient light sources.

Methods Firstly, this paper explores the criteria for healthy lighting based on visual anatomy and pathophysiology and characteristics of workers and tasks in office environments. Secondly, survey methods addressing characteristics of light entering the eye are reviewed in the context of outcomes ranging from discomfort to retinal degradation.

Results A primary consideration is characterisation of light sources in the occupational visual field, (OVF) which is mainly determined by the visual tasks. Another consideration is how, and for how long, the source(s) are imaged on the retina (especially the macula). Again this depends on the visual requirements of the task. Indeed, directionality is much more important for lighting than other hazards such as noise. Such assessments are not possible with an integrating light measuring instrument such as a lux metre. Finally, in the cases of blue-rich sources, the assessment should be more about radiometry than photometry.

Discussion A variety of visual, circadian rhythm and psychological disturbances may arise from inappropriate lighting. The concept of 'healthy' lighting serves to integrate the issues, and surveys of lighting should begin with the OVF and systematic consideration of light sources therein. Assessment should involve luminance and where necessary radiance.

\section{8b PREVALENCE AND RISK FACTORS OF EXTERNAL EYE SYMPTOMS INDOORS}

Peder Wolkoff. National Research Centre for the Working Environment, Lersø Parkallé 105, 2100 Copenhagen, Denmark

\subsection{6/oemed-2018-ICOHabstracts. 1527}

Introduction Eye irritation is generally among top-3 reported symptoms in office environments. The symptoms are especially among middle and advanced ages and particularly among women more than men. The eye symptoms are the most commonly reported complaints in the eye clinic. A multidisciplinary understanding of potential risk factors is necessary to interpret the high prevalence of eye symptoms.

Methods Literature from 2012 to June 2017 has been updated in context of dry eye (DE) symptom reporting in office-like environments by searches in the public databases and previous reviews. 